\title{
Nobel Prize: Facing the Reality of Black Holes
}

\author{
Three scientists were recognized for proving that gravitational collapse \\ can lead to a black hole and for observing the supermassive black hole at \\ the center of our Galaxy.
}

\author{
By Michael Schirber
}

Updated 8 October 2020

We have grown accustomed to the idea of black holes, but their existence took time to gain acceptance. The Nobel Prize in Physics has been awarded to three scientists who made the idea of black holes easier to swallow. Roger Penrose from the University of Oxford received half of the prize for his theoretical description of how gravitational collapse could lead to a black

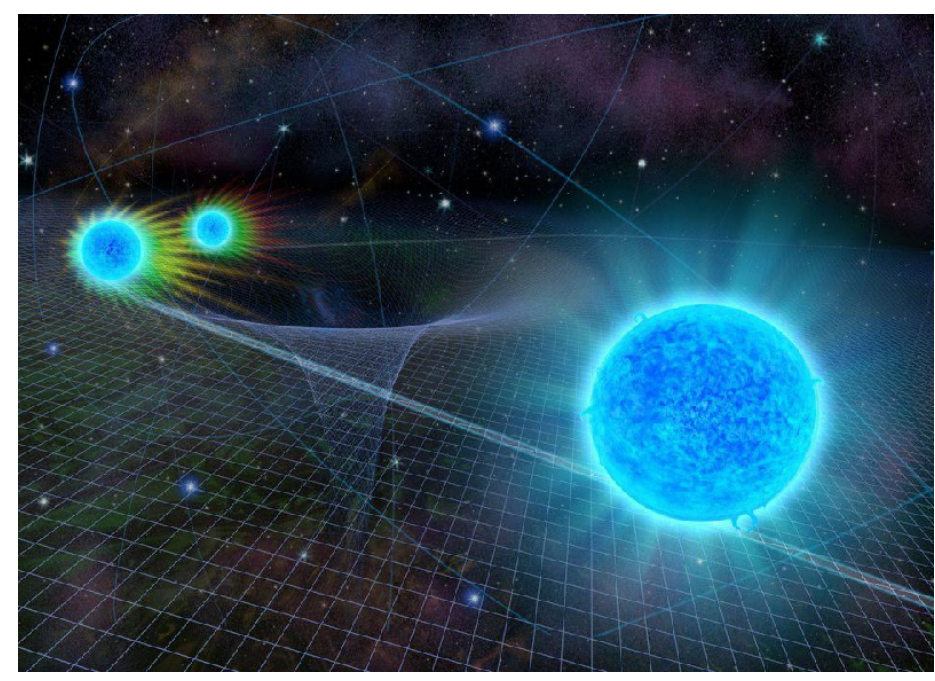

The black hole stage. By observing the path that stars take around the center of our Galaxy, researchers have provided solid evidence for a supermassive black hole. These observations, in addition to earlier theoretical proofs of black hole formation, were recognized by the 2020 Nobel Prize in Physics. (See video below.)

Credit: N. R. Fuller/NSF hole. The other half of the prize recognizes Reinhard Genzel of the Max Planck Institute for Extraterrestrial Physics in Germany and Andrea Ghez from the University of California, Los Angeles, for their efforts in uncovering the supermassive black hole at the center of our Galaxy. Added to recent detections of gravitational waves from black hole mergers, the reality of black holes has become inescapable.

The theory that an object might be dense enough to trap light is more than 200 years old. But the possibility led to something of a crisis in the years following Albert Einstein's publication of his general theory of relativity in 1915 . According to the theory, as a spherically symmetric object collapses, there is a radius (the so-called Schwarzschild radius) at which the infalling material would become cut off from outside observers [1] (see also Focus: Landmarks-Forgotten Black Hole Birth). Even weirder than this "event horizon" was the prediction that a point of infinite density would develop in the object's interior-a kind of big bang in reverse. This so-called singularity seemed so absurd-a potential "failure" of general relativity-that many physicists assumed that some mechanism must prevent a star or other object from ever collapsing that far.

The situation shifted in the early 1960s, when astronomers identified the first quasars-extremely bright objects in distant galaxies [2]. Their enormous energy and small size led to speculations that they might be million-solar-mass black holes (although the term "black hole" wasn't coined until 1967). To make black holes seem more reasonable, however, one needed to show that material can collapse to a point in real-world conditions-that is, without assuming perfect spherical 
symmetry, as previous calculations had done.

Penrose addressed the question of non-spherical collapse in 1965-fifty years after the debut of general relativity [3]. He attacked the problem by arguing that any object with an event horizon-regardless of its shape-will contain what he called a trapped surface. He defined this surface to have the property that, if you placed light bulbs on it, all of the emitted light rays would converge, thanks to the strong gravity. For a sphere, this property implies that all of the light shines inward. The implication is that anything inside a black hole has only one future ahead of it: falling farther toward the center. "Penrose showed that the collapse process can't halt," says gravity expert David Garfinkle from Oakland University in Michigan. "It keeps on proceeding until something catastrophic happens." That catastrophe is a singularity.

Penrose's proof that the collapse of dying stars could naturally lead to singularities was a blow to Einstein's theory, says theorist Vitor Cardoso from the Technical University of Lisbon, Portugal. However, Penrose did not want these singularities to be accessible from outside the black hole. As Cardoso explains, Penrose later developed the idea of "cosmic censorship," in which the black hole's horizon protects external observers from ever observing its singularity (see Synopsis: Cosmic Nudity). Penrose was also an early advocate for observing the skies in search of black-hole-influenced phenomena. "I only wish to make a plea for 'black holes' to be taken seriously and their consequences to be explored in full detail," he wrote in 1969 [4].

Genzel and Ghez took the consequences of black holes seriously. For nearly three decades, they each led teams that collected evidence for a large black hole at the center of the Galaxy. Astrophysicists had previously suspected that such an object might be lurking there, based on the assumed presence of supermassive black holes in galaxies hosting quasars. But there was no direct evidence of a central black hole in our Galaxy.

To build a case for the black hole, the teams began campaigns in the 1990s to observe stellar orbits in the Milky Way's central region-programs that remain active today. Using large telescopes in Chile and Hawaii, they resolved single stars in this very crowded, dust-filled environment. The observed motion of these stars implied a gravitational anchor of four million solar

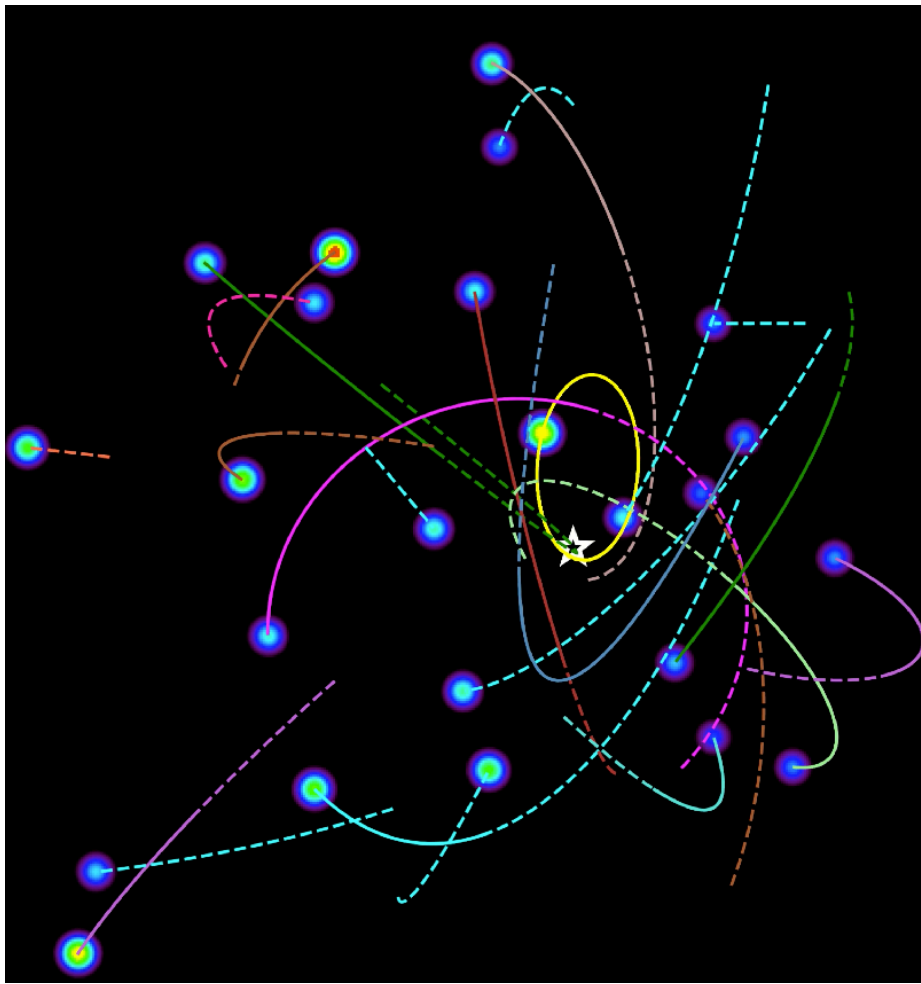

This animation shows stellar orbits in the Milky Way center. The paths imply that the stars are gravitationally bound to a supermassive black hole with a mass four million times that of the Sun. The data were collected by Andrea Ghez from the University of California, Los Angeles, and her colleagues from 1995 through 2020. The star S0-2 is of special interest because it completes a full orbit in about 16 years. (The vertical arrow represents 0.24 seconds of arc, or less than $10^{-4}$ degrees across the sky.)

Credit: Keck/UCLA Galactic Center Group

masses crammed into a region no larger than our Solar System. A black hole was the most reasonable interpretation. "The evidence is so overwhelming that a black hole is the least exotic explanation for the object sitting there," Cardoso says.

The results of Penrose, Genzel, and Ghez are "clearly fantastic discoveries," says theorist Eva Silverstein from Stanford University. She believes that Penrose's work challenged gravitational researchers to go beyond general relativity to explain the interior of a black hole. "The universality of singularities in general relativity, including those inside black holes, captures essential gravitational dynamics while 
demanding a more complete theory of gravity," she says.

Cardoso sees a connection between this year's Nobel Prize and the 2017 prize awarded for the detection of gravitational waves. "These are truly fascinating times for those trying to understand how gravity and the cosmos work," he says.

Michael Schirber is a Corresponding Editor for Physics based in Lyon, France.

\section{REFERENCES}

1. J. R. Oppenheimer and H. Snyder, "On continued gravitational contraction," Phys. Rev. 56, 455 (1939).

2. M. Schmidt, " 3 C 273 : A star-like object with large red-shift," Nature 197, 1040 (1963).

3. R. Penrose, "Gravitational collapse and space-time singularities," Phys. Rev. Lett. 14, 57 (1965).

4. R. Penrose, "Gravitational collapse: The role of general relativity," Riv. Nuovo Cimento 1, 252 (1969). 